\title{
Limitless? Imaginaries of cognitive enhancement and the labouring body
}

\author{
Brian P. Bloomfield Lancaster University, UK \\ Karen Dale $\mathbb{D}$ Lancaster University, UK
}

\author{
Article reuse guidelines: \\ sagepub.com/journals- \\ permissions $\mathrm{DOI}$ : \\ $10.1177 / 0952695119888995$ \\ journals.sagepub.com/home/hhs
}

This article seeks to situate pharmacological cognitive enhancement as part of a broader relationship between cultural understandings of the body-brain and the political economy. It is the body of the worker that forms the intersection of this relationship and through which it comes to be enacted and experienced. In this article, we investigate the imaginaries that both inform and are reproduced by representations of pharmacological cognitive enhancement, drawing on cultural sources such as newspaper articles and films, policy documents, and pharmaceutical marketing material to illustrate our argument. Through analysis of these diverse cultural sources, we argue that the use of pharmaceuticals has come to be seen not only as a way to manage our brains, but through this as a means to manage our productive selves, and thereby to better manage the economy. We develop three analytical themes. First, we consider the cultural representations of the brain in connection with the idea of plasticity - captured most graphically in images of morphing - and the representation of enhancement as a desirable, inevitable, and almost painless process in which the mind-brain realizes its full potential and asserts its will over matter. Following this, we explore the social value accorded to productive employment and the contemporary (biopolitical) ethos of working on or managing oneself, particularly in respect of improving one's productive performance through cognitive enhancement. Developing this, we elaborate a third theme by looking at the moulding of the worker's productive body-brain in relation to the demands of the economic system.

Corresponding author:

Karen Dale, Lancaster University, Department of Organisation, Work and Technology, Bailrigg, Lancaster LA1 4YX, UK.

Email: k.dale@lancaster.ac.uk

Keywords

biopolitics, cognitive enhancement, imaginaries, 'smart drugs', the productive body 
'The average person uses $10 \%$ of their brain capacity. Imagine what she could do with $100 \%$ ' -tagline for the film Lucy (2014)

'Everything is possible when you open your mind'

-tagline for the film Limitless (2011)

\section{Introduction}

Papadopoulos (2011: 432) contends that 'every epoch has its brain' and that the concept of brain plasticity 'occupies the brain-body imaginary of the contemporary epoch'. ${ }^{1}$ The idea that the capacity of the brain is not fixed, that it is an organ with the potential to adapt and change, underpins and finds expression in the current scientific research and wider public interest in pharmacological cognitive enhancement. The possibility of increasing 'brain power' through pharmaceuticals - sometimes referred to colloquially as 'smart drugs' - has gained considerable prominence in popular culture, science magazines, and the wider media, as well as in policy debates. ${ }^{2}$ For example, in recent mainstream big-budget films such as Lucy (2014) and Limitless (2011), drugs with potent powers of enhancement enable the central characters to overcome the limitations of the 'normal' human brain and thereby exert their influence on the world around them. In a more modest vein, media reports have discussed 'normal' people taking drugs believed to enhance cognition in the context of employment, including speculation about 'How Smart Drugs and Cybernetics Could Create a Superhuman Workforce' (Louv, 2012; see also 'The Pharmaceutical Path to a Superhuman Workforce', 2012). At the same time, a number of prominent policy-orientated reports have discussed the possible economic and social benefits of cognitive enhancement drugs (see, for example, Academy of Medical Sciences, 2012; British Medical Association, 2007).

Notwithstanding the distortions of the scientific concept of brain plasticity within its popular manifestations in the media and popular culture, the idea that drugs have the power to enhance the brain or unlock its potential is consistent with a general turn to pharmaceuticals to solve a whole range of problems and achieve desirable ends for individuals and society - 'a pill for every ill' (Beaconsfield, 1980). ${ }^{3}$ This tendency has been described as 'pharmaceuticalization' (Abraham, 2010; Busfield, 2010). It is also expressive of the prevalent view of brain functioning as essentially constituted by neurochemical processes and interactions, which themselves can be adjusted and readjusted through the use of pharmaceuticals. This 'psychopharmacological imaginary' (Rose and Abi-Rached, 2013: 12) appears to hold out the promise to help people manage not only specific diseases of brain function, but also, importantly, aspects of ordinary everyday life (see, for instance, Fox and Ward, 2008; Williams et al., 2008). But at the same time as greater control seems to be available through pharmacology, Rose (2007) argues that the more we see ourselves in terms of brain chemistry, the more we become subject to neurochemical evaluation and intervention.

Discussion of cognitive enhancement falls broadly into two areas: bioethical debate and sociological studies. Ethical discussions centre on two key issues. First, coercion versus free choice: whether individuals might seek to enhance themselves out of their 
own volition, or because they may be required to enhance, or might feel pressured to do so, due to working conditions or to keep up with (enhanced) others in education and the labour market. The pressures for productivity or profitability, the impetus to reduce the costs of labour, and the current move towards more casualized employment conditions are some of the main drivers that could lead to coercion to enhance. Ethical debates tend to problematize coercion, but do not question what is assumed to be its opposite: 'free choice'. Analysing the context of the contemporary labour market and employment relations enables an understanding of the conditions under which this 'choice' comes to be seen by individual employees or students as possible or desirable. Second, there is the question of fairness/equity in the access to such drugs and their outcomes: whether consumption of enhancement drugs might give an unfair advantage to some people who can afford them over others who cannot (Farah et al., 2004; Greely et al., 2008).

Sociologically orientated work on cognitive enhancement has tended to use it to develop explorations of the concepts of medicalization, biomedicalization (Coveney, Gabe, and Williams, 2011) and pharmaceuticalization (Williams et al., 2008). Although this is not the focus of these studies, they have made some points on specific political economic aspects of cognitive enhancement. In particular, this has been discussed in relation to the management of sleep and how the 'customisation' and even potential 'optionalisation' of sleep provides opportunities for greater productivity, especially in the light of shift work (Coveney, 2011; Williams, Coveney, and Gabe, 2013; WolfMeyer, 2012). Other research has considered enhancement drugs in relation to the pressures associated with the characteristics of contemporary employment - including increasing demands for flexible labour, precarity, extreme forms of working, long working hours, 24/7 availability, and so on (see, for instance, Bloomfield and Dale, 2015; Smith and Land, 2014).

Whilst acknowledging the importance of the issues addressed in such literature, here we seek to situate cognitive enhancement as part of a broader relationship between cultural understandings of the body-brain and the political economy. It is the body of the worker that forms the intersection of this relationship and through which it comes to be enacted and experienced. Through our analysis below, we argue that the use of pharmaceuticals has come to be seen not only as a way to manage our brains, but through this as a means to manage our productive selves, and thereby to better manage the economy. More specifically, in this article we investigate the imaginaries that both inform and are reproduced by representations of pharmacological cognitive enhancement, drawing on cultural sources such as newspaper articles and films, as well as policy documents and pharmaceutical marketing material, to illustrate our argument. Previous studies have analysed media reporting on drugs such as modafinil (Coveney, Nerlich, and Martin, 2009; Williams et al., 2008), or the portrayal of a range of enhancement technologies in science fiction (Delgado et al., 2012). However, in this article we analyse a range of cultural sources, arguing that despite their differences, they also encapsulate a commonality in their construction of images of minds and brains, and their potential for enhancement. As the basis for our analysis, we contend that prevalent representations of 
cognitive enhancement are inextricably intertwined with the contemporary social context. As Hogle argues,

The work that goes into both identifying and amplifying certain characteristics as being amenable to change and constructing certain traits as desirable does more than essentialize them as preferred human traits. Rather, it forms a circuit of enterprise, biology, medicine, and culture in complex relations to each other. In this sense, the traits being enhanced are not inherently natural but cultural. (Hogle, 2005: 703)

We contend that the cognitive traits that are associated with pharmacological enhancement are predominantly concerned with making the body more productive and thus linked to particular characteristics that are seen as having economic worth, and thereby connected to the broader political economic context.

Enhancement for the purposes of improving work rates is not new. Historical examples of pharmacological modification of cognition include the military deployment of amphetamines and methamphetamine to improve attention and wakefulness during the Second World War (Bloomfield and Dale, 2015; Rasmussen, 2008). We can see this as part and parcel of the longstanding relationship between the economy and the body. This is captured in Foucault's remark that 'in fact the two processes - the accumulation of men and the accumulation of capital - cannot be separated' (Foucault, 1977: 221). Whilst the human body is the primary instrument of labour, it is also a significant limitation on it. For some time now, the economy has demanded flexibility on the part of the labouring body (Martin, 1994), and in the current era of brain plasticity this translates into the idea of enhancing cognitive powers. Cognitive enhancement may thus be seen as a form of work on the body - an accumulation strategy (Harvey, 1998; Harvey and Haraway, 1995) - that aims to reshape it to fit the particular demands of the economy. Indeed, it is very noticeable that discussions in the news media of the potential for cognitive enhancement through the use of 'smart drugs' frequently focus on how this might improve various working or studying practices. Modafinil, a stimulant synthesized in the 1970s in the context of brain research and sleep, is medically prescribed for conditions such as narcolepsy. However, it has been deployed by various military forces (Moreno, 2008) and subsequently taken up within wider society as a cognitive enhancer, a 'smart drug'. For example, in 2011 Reuters Health reported on research into whether 'the sleepfighting medication modafinil may boost the brain power of weary surgeons' (Joelving, 2011). Similarly, other coverage suggests that enhancement drugs might improve driver performance and safety (Diver, 2017; Margo, 2000). In this guise, modafinil has also proved particularly popular amongst students (see, for example, Dietz, Soyka, and Franke, 2016) and others in high-pressure occupations, even though its efficacy remains a subject of scientific debate (see, for instance, Repantis et al., 2010), along with concerns about its known side effects.

Paid work is multifaceted. It is about more than production or making a living. In capitalist economies, paid work is a source of social status, social interaction, and social identity formation. It has been argued that more and more social relations come to be 
viewed through the lens of economic worth, such that being 'productive' is socially valued and validated whilst there is a concomitant demonizing of those who are 'unproductive' (Fleming, 2015; Smith and Riach, 2016). More recently, conditions of employment have become framed within the language of what constitutes neoliberalism. Mindful of the ubiquitous but often ill-defined use of the term (Flew, 2014; Lemke, 2002), we limit our reference to neoliberalism specifically to the forms of governmentality articulated by Foucault in his lectures of 1978-9. Here he elucidates the development of ideas and institutions that promulgate the 'generalization of the economic form of the market...throughout the social body' (Foucault, 2008: 243) Foucault analyses neoliberal developments in relation to biopolitics: the ways in which life itself is 'put to work'. In this conception, where each individual might be seen as a 'micro-enterprise', investing in themselves to gain the best return on their own self as 'human capital', it becomes more comprehensible why some people might choose to, or feel they need to, turn to smart drugs and other technological interventions in order to succeed, compete, or even just survive in this 'enterprise society' (ibid.: 226). With the growth of neoliberal economics, the focus has shifted to the responsibility of the individual to work on their own body and, we would add, their brain. This then becomes part of an individual project of self-construction - an 'ethic of personal self-care and responsibility linked to modifying the body' (Pitts-Taylor, 2010: 639) - whilst remaining tied to the wider political economy. It is in this context that we would note there is a continuity between students taking cognitive enhancement drugs as a study aid and employees taking them to perform better at work. As one prominent headline put it, 'Students Used to Take Drugs to Get High. Now They Take Them to Get Higher Grades' (Cadwalladr, 2015; cf. Williams et al., 2008). Within the context of neoliberal biopolitics articulated by Foucault, there is a subtle shift where education becomes one of the means by which individuals can improve and actualize their 'human capital', postponing earning opportunities in the present in order to invest in their future employability and earnings potential (Foucault, 2008: 228-30). In the light of this, the consumption of pharmaceuticals for cognitive enhancement can be seen as a potential tool for the worker acting as an 'entrepreneur of the self' (ibid.: 226). One example of pharmacological enhancement that perhaps particularly well illustrates its interrelationship with contemporary employment is the renewed interest in lysergic acid diethylamide (LSD) used in very small quantities as a potential spur to creativity, especially in the context of occupations such as software engineering (Karim, 2017; Kuchler, 2017). In conditions of uncertainty and competition over jobs, even for those in the professions, including the much-discussed replacement of human workers with robots and AI and the casualization of workers' contracts and rights in the so-called 'gig economy', workers seek for the means to reduce their precarity and increase their 'competitive edge'. In contemporary 'knowledge-based economies', it is the 'gold in workers' heads' that is particularly valued, including such skills as creativity and innovation. Thus it is not surprising to find enhancement practices focused on these traits.

In summary, cognitive enhancement resonates with the prevailing political economic order, in particular its valuation of productive performance and the associated 
expectations on individuals to take responsibility for realizing their own potential in order to achieve this. We contend that this ethos produces a commonality that runs through the seemingly diverse representations of cognitive enhancement that we analyse in this paper, ranging from fantasies based upon the acquisition of superhuman skills, through cognitive enhancement, to more 'mundane' pharmaceutical interventions aimed at managing cognitive functions such as alertness and attentiveness, as well as attempts to attain greater focus and improved memory.

\section{Imaginaries of cognitive enhancement}

In order to further explore how cognitive enhancement is represented and understood in everyday life, we deploy the analytical term imaginaries as a means of highlighting the connection between ideas, imagery, and context (on this, see for example Bloomfield and Doolin, 2011; Le Doeuff, 1989; Macnaghten, Kearnes, and Wynne, 2005; Taylor, 2002). Imaginaries relate in part to the cultural images and ideas that circulate, as well as to the various ways in which people relate to them by interpretation, incorporation, and rejection, often contradictorily, sometimes explicitly, and sometimes without intention or conscious deliberation. Therefore, we purposefully refer to 'imaginaries' in the plural not the singular. The imaginaries we explore express contestations and struggles surrounding cognitive enhancement. We do not consider here how these imaginaries of enhancement are received - it is enough to note that we do not see them as deterministic or predictable (O'Connor and Joffe, 2015; Racine and Forlini, 2010). Instead, we aim to elaborate specific aspects of the social, cultural, and economic context from which they emerge, and which they in turn help reproduce.

The imaginaries of cognitive enhancement that are explored in this paper are not passively derived from current dominant images in society, nor are they simply abstract fantasies of how society might be different: They stand in the interstices between such images and those future states (Dawney, 2011; Gatens, 1996). Imaginaries have material effects; they are intrinsic to the possibilities of action, because they hold out the prospect of a future path for the individual and thus motivate desire and choices. Dawney's (2011: 538) development of the concept of imaginaries as 'material, embodied and affective' takes this further:

Ideas and imaginings do not cause practice: they are practices. In other words, to position the imagination in the realm of ideas alone runs the risk of excluding a consideration of the immediate, sensate and embodied modes through which imaginaries come to be experienced and felt. (ibid.: 539)

However, imaginaries have a material effect not only for individuals, but also through how they come to frame future possibilities through cultural and scientific understandings. For example, in 1628 William Harvey described the circulation of the blood through the pumping motion of the heart. Since then, the major imaginary of the heart as a functional organ has treated it as if it is a pump, even though this ignores 
important aspects of its electro-biochemical characteristics. In the 20th century, doctors worked out how to replace a faulty heart with a device that was indeed very like an electrical pump (Laurance, 1995; Dale, 2001: 94-5; Sawday, 1995: 31).

Our analysis below shows that the contemporary imaginaries of cognitive enhancement present in a diversity of sources are significantly embedded in cultural constructions of what might be described as 'the productive body' (Gue'ry and Deleule, 2014): that body which is made fit for work and employment. However, beyond the immediate demands of the working body, these imaginaries express aspirations for performance that are characterized by an increased emphasis on achievement, personal development, and realizing one's potential.

The article draws upon a variety of English-language sources of material that refer to pharmacological cognitive enhancement deriving from the period 1997 to 2017. The selection of a range of sources was shaped to a certain degree by our awareness of the interplay or cross-referencing between them. Along with the growing media (including Internet) coverage and public discourse surrounding the topic of cognitive enhancement, and with its manifestation in popular culture through films such as Limitless and Lucy, it was interesting to observe that the latter became drawn upon in those media reports as a means of narrating the topic to their audience. Furthermore, informed by other research on drugs and the brain that examined the role of industry advertisements (see, for instance, Singh, 2007; Tone, 2009), we too chose to consider the marketing materials for the drug modafinil. We view our sources as related cultural or social manifestations of the notion of brain plasticity in general, and the imaginaries of cognitive enhancement in particular. In short, we regard our chosen sources as 'public fragments of social consciousness that work (albeit loosely) in concert, encouraging people to reason, know and fashion their worlds in particular ways' (Kroll-Smith, 2003: 627). Accepting this commonality, it is nonetheless useful to distinguish the particular characteristics of the sources, as they each have different relations with the portrayal of the enhanced or modified brain in everyday life.

The first category of sources comprises portrayals of cognitive enhancement in popular culture, specifically the recent films Limitless and Lucy. Although explicitly fictionalized narratives expressing fantasies far from everyday experience and current possibilities, they invoke distorted or exaggerated (pseudo)scientific ideas about the brain and the possibilities of pharmacological enhancement, and in doing so tap into some key desires and anxieties about its implications.

Second, we draw upon international English-language media reports of pharmacological cognitive enhancement, particularly newspaper articles. Whilst at one level, some news coverage purports to present factual accounts of scientific developments in brain science, neuroenhancement, or the use of 'smart drugs' amongst particular groups in society, it is also involved in the construction of particular imaginaries about such matters. Indeed, since there are currently no drugs that are licensed to be prescribed or marketed as cognitive enhancers, these reports are inherently involved with the formation of narratives of what these 'smart drugs' are and how they are used (see Kroll-Smith, 2003). Such accounts typically refer to the off-label use of 
prescription drugs such as modafinil and Ritalin, usually associated with diagnoses such as narcolepsy and attention deficit hyperactivity disorder (ADHD), respectively, but consumed (without a medical prescription) by people who have not been given these diagnoses, with the aim of improving cognitive function. Scientific studies are mixed in their conclusions as to whether brain functioning is improved, and in any case laboratory studies are hardly representative of everyday work life. Overall, the drugs predominantly promote wakefulness rather than increase cognitive ability. Nevertheless, many media accounts relate the (supposed) improvement in brain ability to the possibilities of, or at least desire for, increased performance - such as in study or at work - and thus the pursuit of self-development promoted by neoliberal discourse. For instance, taking a snapshot of coverage in UK national newspapers in 2016 revealed 20 unique reports on the topic of modafinil or smart drugs and the brain, of which 18 referred to enhancement (positively or negatively) in the context of performance in study or at work. ${ }^{4}$ We contend that such examples draw upon particular ideas and social values - imaginaries - surrounding selfrealization, employment, and the social valuation of productive effort (characteristics associated with neoliberalism), but at the same time offer imaginaries of cognitive enhancement that in turn reproduce those ideas and values.

The third category we draw upon is marketing material for the drug modafinil (and its variants). We chose to concentrate on modafinil, rather than other substances that are discussed as enhancers, for several reasons. First, it is the most common substance referred to in media reports. Second, there has already been a more formal crossover of the drug into work environments: It has been used in the military and discussed in relation to long-distance driving as a potential aid to safety, and experiments with it taken place in the medical and surgical field (Krueger and Leaman, 2011; Sugden et al., 2012; see also Bloomfield and Dale, 2015). Third, there was a meta-analysis of scientific studies of modafinil in 2015 (Battleday and Brem, 2015), which led to its being labelled 'the world's first safe smart drug' in newspaper reports (Thomson, 2015), thereby increasing its visibility. And fourth, the advertisements for modafinil specifically relate its use to employment. Official promotional material from the pharmaceutical industry does not represent cognitive enhancement as such, since it is not allowed to market drugs for anything other than their licensed uses. ${ }^{5}$ However, we contend that the adverts nonetheless offer imaginaries about the relationship between the brain and potential pharmaceutical interventions in its functioning; for instance, in terms of restoring alertness, attention, or wakefulness in sleep-deprived individuals. The imaginaries typically deployed in promoting a drug refer to both its power to transform an individual's condition and the future self that they hope to become. One of the ways in which industry seeks to convey product information to consumers is through the use of narrative devices and associated imagery centred on clearly recognizable as well as believable characters; individuals that one can identify with. In this regard, the promotional material for pharmacological drugs is often no different (see Frosch et al., 2007; Rasmussen, 2008; Singh, 2007; Tone, 2009). Such material and its associated imaginaries present possible identities that the observer can 'try on' to see if they would fit into the imagined future that is portrayed therein. The envisaged use of the product that is being promoted may 
thus become thinkable as a first step towards its actually being acquired. Moreover, imagined futures have an emotional component, and this is exploited in a number of advertising campaigns for various pharmaceutical drugs (Frosch et al., 2007).

There is also unofficial, unregulated marketing material from online retailers who overtly promote the supposed cognitive enhancement potential of these drugs, with some making an explicit link to the Limitless film, offering the 'real' smart drug. This material includes narratives that are presented as the experiences of those who have tried the drugs (though obviously this cannot be verified, and the experiences are clearly portrayed in a particular way, since they are made available on sites designed to promote the sales of the drugs). It also includes online discussions between users, and other information that is presented as factual about the drugs.

Fourth, we draw upon two policy reports that have been published in the UK on the use of drugs for the purposes of enhancement: the Academy of Medical Sciences 2012 report on Human Enhancement and the Future of Work, and the British Medical Association's 2007 publication Boosting Your Brainpower: Ethical Aspects of Cognitive Enhancement. These are relevant because they bring in discussions that cut across the scientific and policy communities and seek to construct future-orientated activity, especially with regard to the economy.

As we have already noted, despite the seeming diversity of these materials, it is the strands of commonality between them that enable us to better understand the ways in which the associated representations of the brain relate to the cultural context out of which imaginaries of enhancement emerge as immanent potentialities. This can be shown by briefly illustrating the interrelationships between the different sources. For example, newspaper reports pick up on scientific and policy discussions that they then re-present in a popular, digestible form. Similarly, references to 'the real life "Limitless" drug' can be found in newspaper headlines and online pharmacies for modafinil; and reviews of the film claim that it was based upon modafinil. ${ }^{6}$

Our main argument in what follows is structured according to three analytical themes followed by a concluding discussion. In the first of these, Mind over matter?, we explore the commonplace imagery of the brain that is deployed in popular coverage of enhancement. The second theme, Valuing productivity and performance, examines the connection between imaginaries of enhancement and the social valuing of productivity and performance, especially in paid employment. The third theme, Enhancing the economy, considers how management of the (neoliberal) self is but a microcosm of broader managerial efforts to organize the world. Noting Wolf-Meyer's (2009: 13) point about the 'need to understand the economy as an always embodied practice', we illustrate this theme by reference to efforts to exercise pharmacological control over alertness, wakefulness, and sleep.

\section{Mind over matter?}

In the film Lucy, we see portrayed a fantasy of total control in which the mind-brain has power not just over the individual's body, but the external world too. For example, Lucy 
is instantly able to accomplish complex tasks involving adept physical coordination by the sheer power of thought/knowledge. At the beginning of the film she is unable to drive, but once the drug has enhanced her brain she transforms into someone who can skilfully weave a car through fast oncoming traffic. Similarly, through the power of the drug Lucy can immediately understand languages that she could not previously speak, and is able to wield a gun like a professional. The embodied nature of human skill acquisition and practice is ignored: Her new abilities are derived internally, as it were, directly from her brain power - she just knows what to do and is able to do it. This portrayal of the possibilities of cognitive enhancement sidesteps any understanding of how learning comes from embodied interaction with the world. Moreover, as Lucy's powers develop she becomes able to exercise telekinetic and other powers over matter itself. Everything becomes subject to her will, which can be perfectly enacted because of the realization of the full potential of her brain.

Although easily dismissed as science fiction fantasy, Lucy may be better understood as the extension of current lines of thinking pushed to their limits. This imaginary of cognitive enhancement shows both connection with and contrast to the current dominant 'materialist' view of the mind-brain, where mind is understood as a property of the brain, which is an organ of the body (Rose and Abi-Rached, 2013: 1). Here, on the one hand, we see the brain as an organ of the body whose biological capacity can be extended through pharmaceutical substances. On the other hand, it is seen as capable of being enhanced as if it was autonomous and almost separate from the body, in a way that is suggestive of a power of mind that goes beyond its biological nature. This evokes images of an enhanced brain, which is now able to 'pull along' a body that can be perceived as a constraining factor due to its biological limits. Lucy's body before it is enhanced is weak and deficient - she is not able to resist those who forcibly turn her into a drugs mule. Once she is at the full extent of her extraordinary pharmaceutical enhancement, however, her brain becomes all-powerful - indeed, so much so that the biological body is no longer able to contain it, and she becomes a supercomputer before dissolving and leaving her superior knowledge behind on a flash drive. In this strange sequence, we see the dream of overcoming and even entirely transcending the physical body, a fantasy that resonates with a long history of denial and degradation of the body (Turner, 1984). We suggest that there is a residual Cartesianism in this imaginary, which coexists and is in tension with the prevalent materialist view.

Within more mundane everyday examples of imaginaries around cognitive enhancement we can see similar themes. Newspaper reports about the use of 'smart drugs' emphasize how they provide a means to transcend biological limits. Headlines include "In the City That Never Sleeps...Traders Stay Up on "Smart Drugs" (Dean, 2013), 'Public Servants Used Drug, Modafinil, to Stay Awake to Complete the Federal Budget on Time' (Farr, 2014), 'Drug-Taking: Think What We'd Achieve If We Never Slept a Wink' (Clay, 2012), and 'Smart Drug Helps You to Sleep Less and Think More' (Lay, 2015). All of these examples rely on an imaginary where the limits of the tired body can be overcome by an enhanced brain. The visual imagery that is often deployed next to articles such as these is also telling: for example, a brain with coloured lines radiating out 
of it, and a brain with sections brightly lit up (Petrow, 2013; Lay, 2015). Again, a notable common theme with these pictures is that the brain is shown as a single self-contained organ abstracted from the rest of the body; it appears to be able to exist and function alone and independently, as a disembodied agent. This somewhat mechanistic view of the brain runs through much of the discussion and visual representations of cognitive enhancement.

The problematizing of the body is something that can also be discerned in the official adverts for Provigil (an early tradename for modafinil) in the USA. These directly promote the use of the drug for patients reporting symptoms of excessive sleepiness (ES). For example, a dramatic transformation from sleepiness to alertness is captured explicitly in an advert for Provigil that appeared in professional journals such as Psychiatric News. ${ }^{7}$ Under the caption 'Cut through the fog of ES with PROVIGIL', we see an image of a female clinician in the foreground who is striding ahead, bright and alert. In the background lurk several other figures, all bearing the trappings of their employment, but shrouded in fog and looking tired and deadbeat from their work. A series of related images in other adverts similarly portray the 'before' and 'after' message, with the former depicting tired, aching bodies and downcast eyes, and the latter showing figures who after taking modafinil - are energetic, refreshed, and committed to their work. The suggestion that is carried through this promotional material is one that poses the chemically enhanced brain as a 'solution' to the failing body. As Elliott (2003: 13) notes, 'Enhancement technologies are usually marketed and sold by taking advantage of a person's perception that she is deficient in some way'.

The tensions that we see in the imaginaries of cognitive enhancement illustrate the complex and continuing power of Cartesianism, with its mind-body dualism, and hierarchical assumptions that the mind constitutes the active subject whilst the body is mere matter. The 'brain' in some ways occupies an ambiguous and ambivalent position: Sometimes it is equated with the power of mind (over that of matter), and sometimes it is equated with the limitations of that biological matter (as an organ of the body). Furthermore, despite the prevalent idea that the mind is now embodied in the brain, which is another organ of the body, there is an assumption that enhancing the mind-brain can be secured without risk or detriment to the passive body, with 'side effects' considerably downplayed.

The transformations of the body-brain depicted in films such as Limitless and Lucy, as well as in the adverts discussed above and in cultural representations of enhancement more generally, can be further analysed in terms of Sobchack's (2000) discussion of 'morphing'. This is a technique deployed in cinematic representations of radical bodily transformation. These metamorphoses depict bodily changes without any of the 'natural' biological processes involved; they are about 'making visible (and seemingly effortless) incredible alterations of an unprecedented plastic and elastic human body' (ibid.: 45). In doing this, they ignore, obscure, or even write out the time and pain involved in the experience of bodily change: 'transformation without time, without effort, without cost' (ibid.: 50). For example, in Limitless radical cognitive enhancement is achieved (and sustained) through taking a fictional drug, 'NZT-48'. In this case, biological processes 
and time are doubly removed. First, because once a pill is consumed it moves from the outside to the inside of the body, and the changes it instigates themselves become invisible and no longer consciously thought of (Martin, 2006). Second, because the (imagined) changes of enhancement are refracted through the remnants of the Cartesian body. Thus, the dominant imaginary runs, these drugs produce specifically cognitive enhancement. Aside from the resultant powers that are bestowed by the pill (an ability to play the piano or speak other languages inevitably requires the body to behave differently, even if this is not acknowledged), the only sustained bodily changes that we see are relayed by a pronounced brightening of Eddie Morra's (the central character) irises. They seem to radiate, indexing cognitive prowess. Dramatic negative effects are visited on the body, but only as a consequence of the drug wearing off, the effects being quickly reversed once the drug is consumed again. There is thus an asymmetry as regards bodily processes: a seamless, almost disembodied transition to enhanced powers through the presence of the drug, and then a body rendered lumpen and dazed by its absence.

The plasticity that is assumed and represented in morphing techniques can be related to the dominant image of the plasticity of the brain. But as Sobchack (2000: 45) notes, this plasticity comes with connotations and consequences for embodiment and social relations, 'rendering human affective states with unprecedented superficiality and literalism'. At the same time, 'the plasticity of the image (and our imagination) has overwhelmed the reality of the flesh and its limits' (ibid.: 50). The bodily nature of the brain, with its biological time and processes, its emotions and interconnections, is effectively written out. Through this, the brain becomes rendered open to commodification and instrumentalism, both for the individual and within the broader political economy. Featherstone (1991) notes that the body in contemporary consumerist culture has become seen and experienced as plastic, and hence a lifestyle accessory, a thing to be sculpted, shaped, and 'stylized'. Similarly, Emily Martin develops this theme in Flexible Bodies, here placing it in an economic context, and arguing that 'flexibility is an object of desire for nearly everyone's personality, body and organisation' (Martin, 1994: xvii).

In the next section, we further develop our analysis by discussing how the impetus to overcome the limitations of the body-brain through cognitive enhancement is socially and morally legitimated in relation to the imperative to be an economically active and productive subject.

\section{Valuing productivity and performance}

The potential to enhance the brain is linked to the high valuation that capitalist societies put on productivity, and thus on being a productive person. An article in the New Yorker entitled 'Brain Gain: The Underground World of "Neuroenhancing" Drugs' (Talbot, 2009) tells of a Harvard graduate who as a student regularly took Adderall as a study aid. ${ }^{8}$ His summing up of this: 'Productivity is a good thing'. Another account in this article tells of someone who works with a colleague who takes modafinil and, in contrast to them, is seen by their boss as a problem 'for not being as productive'. A third story is 
of an older person experimenting with modafinil, who believes he is 'performing a little better'. The moral of this particular smart drug tale is that productivity is good, and hence achieving it through pharmaceutical means is also good. The rhetoric of productivity, and the expectation that the individual will work on their ability to be productive, legitimates the use of smart drugs.

Imaginaries of cognitive enhancement are closely entwined with cultural norms and values of productivity and performance. At the level of the individual, there is an expectation that in order to legitimately participate within society, one has to be a productive person. For example, this underpins the argument of the Academy of Medical Sciences report Human Enhancement and the Future of Work:

Enhancement could enable more people to work at their full biological capacity and to meet necessary entry requirements for an occupation, which could result in a rise in standards or potentially greater opportunity and diversity at work. Individuals with lower cognitive abilities tend to have less choice of occupations, but enhancement may enable them to compete and thus have greater choice. (Academy of Medical Sciences, 2012: 44)

This articulation indicates a key shift in the contemporary relationship between the individual employee and their place in the labour market. The language is that of choice and competition, but in the sense that the individual, in order to have greater opportunities in their working life, has a 'choice' of enhancing themselves so they can better compete with others. The significance of this can be further seen in imaginaries of cognitive enhancement that find expression in popular culture. One of the straplines for the film Limitless is, 'What if a pill could make you rich and powerful?'. Similarly, newspaper articles and blogs are headed: 'Nootropics [substances that improve mental function]: Can These Smart Drugs Super-Charge Your Career?' ('Nootropics', 2013);' 'Smart' drugs are coming to the office - to make you sharper, stronger...better" (Metro, 1st June 2016); ${ }^{10}$ and 'How (and Why) to Use Nootropics to Boost Productivity and Performance' ('How (and Why) to Use Nootropics', 2016). It is noticeable that these headlines address the individual worker directly.

In this framing, then, there is a sense in which the worker is expected to want to make themselves more employable, in order to achieve their own full potential and selfactualization. From this perspective, it is the worker who has to ensure that they are fit for work - in other words, to 'choose' to enhance themselves and make themselves into a productive body, and brain. In early industrial work, the worker was fitted to the job, in the sense that it was recognized that different workers had different levels of skills and abilities. Techniques were devised to measure these and thereby 'sort' workers into their appropriate places within the labour market (Hollway, 1991). However, in the contemporary world, for many there has been a shift of responsibility towards the individual to make themselves 'employable', to take responsibility for their own wellbeing such that they are 'fit for work' - effectively, to make of themselves a marketable asset that can be sold to the highest bidder in the employment market (Dale, 2012; Dale and Burrell, 2014). 
This elision between productivity as being something that we do 'at work', and productivity being a characteristic that we have or are, can be discerned in entries in blog discussions amongst those who take modafinil/Provigil:

'[Modafinil] saves the day. I work full time, intricate work, drive, multi task person.... I'm the energizer bunny. Patricia Jung, August 13, 2008';

'This turned me from a deep, suicidal depression into a happy, productive person in 2 days....Paul Minor, April 7, 2010';

'At first I only took it when I needed it, but finally I gave up having so many nonfunctional days that I started taking it every day....My days are much more fulfilling.... Bloomer, February 13, 2009'

(Source: modafinilorder.com; emphasis added) ${ }^{11}$

We can link these commentaries on the benefits of the drug in terms of productivity back to the earlier discussion on the need for the flexibility of workers' bodies. The imaginary of the plasticity of the brain, harnessed through the use of cognitive enhancers, becomes yoked to this impetus to continually provide potential for ever-greater performance and productivity. Thus the speculative nature of contemporary capitalism (Cooper, 2011) is worked out through the possibilities of enhancement: 'The Real-Life Limitless Pill? Drug Helps Adults Learn as Fast as Children by Making the Brain More "Elastic" (Woollaston, 2014). Here the plasticity of the brain has its counterpart in the idea of human 'resourcefulness' - the idea that human qualities can be extended and enhanced, that they are not finite or fixed as are other assets:

The working subject is always capable of 'more', of 'becoming better', of learning, creativity, knowledge and 'talent' beyond that which is currently performed. (Costea, Crump, and

Amridis, 2007: 250)

Thus, the sorts of traits that are explicitly valued here in the employee are also those that are targeted through pharmaceutical enhancement. Enhancement drugs therefore do not solely increase the productivity of an individual in quantitative terms, but also enable employees to demonstrate that they are constantly "'switched on", present, alert, creative and enthused' (Fleming, 2015: 67). In other words, employees have to not only be productive, but look productive. Enhancement drugs aid in this because of their potential to increase focus and attention, even where an employee would otherwise be demotivated or uninterested. For example, in one online article we are informed that 'Lucas Baker, a Switzerland-based software engineer with a large tech company, takes nootropics every day. He says it helps him maintain focus, especially on projects he might otherwise put off. "When I find an unpleasant task, I can just power through it," he says' (Roose, 2015).

In analysing the connections between imaginaries of cognitive enhancement and the valuation of production and performance, we can discern the interplay between the enhancement of the worker's body and the wider political economy. Furthermore, 
following Foucault (2008: 242), we can suggest that these imaginaries denote 'an extension of the economy to the entire social field'. In the next section, we move from considering the individual social relations of enhancement to a broader reflection on what this means for a political economy of enhanced brains.

\section{Enhancing the economy}

As we have seen above, imaginaries of cognitive enhancement are closely entwined with the cultural norms and values of productivity and performance, and at the individual level there is an expectation that in order to legitimately participate within society, one has to be a productive person. In this section, we move from considering the individual (enhanced) brain to look at wider aspects of enhancement in relation to Hogle's (2005: 703) point about the 'circuit of enterprise, biology, medicine and culture'. At a collective level, this means that bodies-brains are themselves seen as the source of productivity and performance for society. For example, a policy discussion paper on cognitive enhancement produced by the British Medical Association asserts the connection between the economy and cognition:

An overall increase in cognitive ability in society could also lead to competitive advantages in the cut and thrust world of international trade and commerce. Fukuyama, who vehemently opposes the use of enhancements nevertheless acknowledges that 'a society with higher average intelligence may be wealthier, insofar as productivity correlates with intelligence'.

(British Medical Association, 2007: 18-19)

This fits with a commonly reiterated view that so-called 'advanced' or post-industrial capitalist economies are more dependent on knowledge, and its associated qualities of creativity and innovation, sometimes described as the 'knowledge economy' or labelled 'cognitive capitalism' (Vercellone, 2005).

This impetus can also be seen in the film Limitless, where cognitive enhancement is presented as enabling and extending a number of intellectual capacities - from playing music to writing books - but its particular emphasis is on becoming so smart/cognitively enhanced as to be able to work in and command the world of corporate takeovers and financial markets. Moreover, in Limitless (and also in Lucy), cognitive enhancement enables the mind to 'read' everything that is going on around it, making the world legible to the human brain. As Eddie, the central character in Limitless, explains, 'Everything I had ever read, heard, seen, was now organized and available'. To be able to 'read' the world is to be able to understand it and thereby have control over it, to predict and change it. Ultimately, this is a form of cognitive knowledge and control that is to be put to work in relation to the economy.

Turning from the Hollywood fantasy of enhancement to its more mundane applications in everyday life, a commonality can be discerned in terms of the orientation towards the economy - especially in enabling the organization and management of employment. Although sleep may be regarded as a precondition for health and hence the 
ability to work, it can also be seen as the absence of productive effort, a lack on the part of the body (Crary, 2013; Fleming, 2015; Wolf-Meyer, 2012). Accordingly, scientific efforts to understand sleep/wakefulness are not just aimed at offering treatments for individuals whose lives are plagued by an inability to regulate their patterns of waking/ sleeping, but are also of increasing relevance to organizations and the demands of an economic system that is geared towards $24 / 7$ operation. It is in this context that Williams, Coveney, and Gabe (2013) discuss the desire for the customization of sleep, with the potential ultimately to make it optional.

As mentioned earlier, the drug modafinil has become most touted as a cognitive enhancer, especially since a meta-analysis of it (Battleday and Brem, 2015) led to its being headlined as "the world's first safe "smart drug"' (Thomson, 2015). ${ }^{12}$ However, between its strictly medical use and the accounts of its enhancement properties (it has also been labelled the 'real-life "Limitless" pill' by online pharmacies and news media accounts), its prevalent use is as an everyday regulator of wakefulness. For example, under the headline 'Sleepless in the City', The Times of India carried the subtitle: 'Modafinil, the latest lifestyle drug in Delhi, makes owls out of human nightbirds...the flip side of Working Delhi's graveyard shift' (Sharma, 2004). It is developments such as this - accounts of how modafinil is used to 'enhance' the lives of those who take it, enabling them to cope with the demands of work - that best illustrate the political economy of pharmaceutical enhancement.

In 2004, the pharmaceutical company Cephalon received official clearance from the US Food and Drug Administration (FDA) to promote Provigil (its branded version of the drug) as a means of alleviating excessive sleepiness and promoting wakefulness in connection with shift work disorder (SWD), a condition associated with a significant section of the workforce engaged in long working hours or nightshift work (Kroll-Smith, 2003). ${ }^{13}$ In 2011, Cephalon ran a new promotional campaign for Nuvigil (another variant of modafinil) targeting prescribing clinicians as well as potential consumers suffering from excessive sleepiness associated with shift work. Deploying the caption 'SUP-

PORTING THOSE OF YOU WHO STAY AWAKE FOR THE REST OF US', one image on its website (also reproduced in related promotional material) presented a picture of four individuals (three male and one female): a firefighter, two paramedics/ clinicians, and an emergency services worker. These familiar, respected, and important figures in the community were presented as professionals who make sacrifices on our behalf. Forfeiting what the rest of us enjoy (at least in theory) - that is, a 'normal' night's sleep - they stay awake, responding to whatever emergency situation arises. The narrative here is that the drug enables these sorts of professionals to attain a better state of alertness or wakefulness whilst on duty, remaining vigilant, attentive, and thereby effective despite working at night or for long hours. Importantly, then, these individuals embody significant social values, such as possessing authority to deal with emergency situations, expertise, courage, duty to others, and caregiving. By supporting them in their night-shift work, the drug is also portrayed as upholding those values. Moreover, to the extent that drugs such as modafinil are portrayed as supporting key social values in the context of 
work, such social values are, conversely, 'borrowed' by the adverts to legitimate their usage.

The official Nuvigil webpages and related adverts in professional journals (such as Medical Marketing \& Media or Pharmacy Today) offer a series of 'user' narratives, including that of Jenn, a 32-year-old emergency room nurse dealing with both shift work and the need to take responsibility for her family: 'I'm so tired on my shift that it's hard to do my job'. Moreover, as well as the general occupational information that her presence on the page presents (she appears dressed in a cap and gown and has a stethoscope around her neck), we are provided with some further individual background information. Jenn, we are told, 'sleeps approximately 6.5 hours during the day, waking to run errands and make dinner for the family'. So, in addition to the social values one might typically associate with an ER nurse, Jenn not only makes a sacrifice by working at night (whilst the 'rest of us' sleep), but she also has responsibilities for others, foreshortening her daytime rest to perform domestic duties. The account suggests that to achieve this, she needs to manage the limitations of her own body, and appeals to modafinil as a solution to her problems. The drug offers a means of controlling her alertness/wakefulness, which in turn would allow her to do her job and manage the rest of her life and responsibilities. Once again, we can see an emphasis on the need for individual autonomy and responsibility - in this case over her performance in work and at home - coupled with a contribution to the collective good. ${ }^{14}$

Of course, we all expect round-the-clock (24/7) availability when it comes to the emergency services. However, notably, the Nuvigil advertising campaign was later to include other rather different categories of workers - bartenders, waitresses, DJs, and warehouse staff - indicating a much broader scope of occupations deemed appropriate for the management of wakefulness. Thus, far from being reserved for people working in the essential or emergency services, the drug was available to support people working within all the services that modern consumer society takes for granted. On the one hand, then, the campaign reinforced the meaning and value of work in contemporary society, as we explored earlier. But on the other, it also indicated a managerial effort to realize a world in which employees' wake/sleep cycles are organized according to the demands of production and the provision of services: the desire to create an always-on body.

In terms of imaginaries, what is notable about the official promotional material for modafinil discussed here is how it contrasts with the commonplace media imagery of an enhanced brain, as noted earlier. Instead of a brain or head in effect abstracted from the rest of the body, we have depictions of real-life embodied subjects who have recourse to the drug in order to stay alert and focused on their work. The material does not promise to boost intelligence, but it does offer the prospect of enhancing the brain's ability to cope with working extended hours or at night. This is not therefore an imaginary about becoming mentally exceptional through pharmaceuticals, but rather one that envisages normal working under conditions that would otherwise lead to a deterioration of cognitive abilities. ${ }^{15}$

Finally, another example of the negative impact on work due to the limitations of the body occurs when individuals cross different time zones - namely, the problems of jet 
lag. A number of remedies have been proposed to deal with jet lag, including the prescription and marketing of melatonin (the hormone that regulates sleep and wakefulness). ${ }^{16}$ In 2010, Cephalon applied to the FDA for the approval of Nuvigil as a possible treatment for jet lag, with business travellers being a particular target of interest (Pollack, 2010). From the perspective of the individual business person, the drug might seem attractive if it allowed them to stay alert and thus work effectively whilst crossing time zones, but from a managerial/organizational perspective, this application would help maximize use of human resources. In the event, the approval of the FDA was not forthcoming, but the fact of the application remains significant when considered in terms of the broader political economy of cognitive enhancement examined here. ${ }^{17}$

In summary, whether it is a matter of enhancing the potential to cope with extended working hours, shift work, or business travel across different time zones, modafinil can be viewed as a putative contribution to the flexibility of labour, offering the prospect of suppressing the disorder to the system that the limited body-brain might otherwise precipitate, and thereby supporting the sort of 24/7 temporalities that are scrutinized by scholars such as Crary (2013), Fleming (2015), Williams, Coveney, and Gabe (2013), and Wolf-Meyer (2009).

\section{Conclusion}

Addressing the confluence of scientific ideas about the brain, pharmacological interventions in cognition, popular culture, and everyday life, we have considered imaginaries of cognitive enhancement in relation to three analytical themes. First, we considered the cultural representations of the brain in connection with the idea of plasticity - captured most graphically in images of morphing - and the representation of enhancement as a desirable, inevitable, and almost painless process in which the mindbrain realizes its full potential and asserts its will over matter. Following this, we explored the social value accorded to productive employment and the contemporary (biopolitical) ethos of working on or managing oneself, particularly in respect of improving one's productive performance through cognitive enhancement. In developing this, we looked at the moulding of the worker's productive body-brain in relation to the demands of the economic system. Aiming to build upon previous sociological studies that have researched individuals' motivations for and views about the decision to take cognitive enhancing drugs (Coveney, 2011; Smith and Land, 2014; Vrecko, 2013, 2015), we have sought to connect the individual worker and their labouring body-brain with the contemporary neoliberal biopolitical context. Here, we briefly consider the consequences of these arguments: first in relation to studies in the history of the human sciences, then in relation to the use of the concept of imaginaries and especially how this relates to the remnants of Cartesianism, which we have analysed in imaginaries of cognitive enhancement; and finally, we reflect on some of the implications in relation to the neoliberal working subject.

The sociological approach adopted here has wider implications in terms of the history of the human sciences. Viewing the shaping of the human body in the context of work as 
an accumulation strategy calls for an examination of the configuration or problematic that interlinks research into the body (including the brain) and associated developments in pharmacology; the pharmaceutical industry seeking to develop markets for its products; and the nexus of social, political, and economic factors that play a role in the constitution of problems or goals for which pharmacological or other technological solutions are sought. Seen in broader historical terms, there has of course been a long line of research endeavouring to understand the human body in order to better harness it to productive effort. Examples such as the 19th-century studies of fatigue (Rabinbach, 1992), scientific management (the human body envisaged and managed as an appendage to productive machinery), and the emergence of the field of work psychology (Hollway, 1991) help set current discussions of work and human enhancement in a broader perspective. In this article, we concentrate on the relations between the notions of brain plasticity incorporated in cognitive enhancement, and how this relates to the specific conditions of contemporary employment.

To this end, we contend that imaginaries are of key importance - in terms of the imagined goals of scientific research (such as the search for an "on/off switch" in brain research); the ways in which these ideas are represented and interpreted more broadly in society, promoted, and marketed; and the part they play in the deliberations, sensemaking, and justifications whereby individuals orientate themselves in terms of their future possibilities for action. Accordingly, in this article we have considered how the imaginaries of pharmacological cognitive enhancement reflect and reproduce a number of key aspects of the contemporary cultural, socio-economic, and biopolitical landscape. Prevalent in a diverse array of sources, imaginaries are not mere abstract fantasies, but rather a key part of how individuals orientate themselves to their future possibilities: In order to act upon the world, individuals need to be able to imagine this action and its outcomes. Imaginaries are therefore performative. There is thus an anticipatory and promissory aspect to contemporary imaginaries about the brain that seems to suggest that not only will we be able to better understand ourselves as humans by understanding the brain, but also this knowledge and its associated techniques will enable us to govern ourselves and human affairs in general, in a better way (Rose and Abi-Rached, 2013).

We would argue that through the use of the concept of imaginaries, there is a possibility to move away from dualistic conceptions, such as mind/body, as well as to better understand the implications of such conceptualizations. We have analysed the Cartesian strand that runs through the imaginary of cognitive enhancement and splits cognition or intellect from the (rest of the) body. One consequence is that the focus on the enhanced brain serves to distance not only the individual's body but also the collective, the social body. Erasing the social in this way not only reinforces the individualist subject position of neoliberalism, but also diverts attention away from substantive consideration of the coercive pressures stemming from the policies and conditions of employment that might drive individuals towards cognitive enhancement.

The Cartesian strand that we perceive in imaginaries of cognitive enhancement sees technology as a means of surpassing the biological limitations of the body in order to 
achieve control through the exercise of the mind, along with its associated knowledge and rationality. Both Limitless and Lucy trade on the idea of unlocking the brain's potential through technology in the shape of a pharmacological substance, in the process acquiring power to act upon and hence manage the world, rendering it subject to organization and thereby control. Thus we might argue that the other side of the plasticity of the brain is the increased malleability of the world. In this connection, it is useful to note the emphasis on progress through technology that is a key trope of modern culture. Underlying this is a predominantly humanistic approach to technology as the product of human knowledge to harness the resources of the earth to human desires and designs. Moreover, the liberal view of enhancing the brain as individual 'free' choice is allied to the longstanding North Atlantic/Occidental notion that the human species inevitably seeks to better itself, to improve on the present by embracing the future - what might be seen as the 'ascent of man [sic]' - as depicted in Limitless, or even a move to a posthuman or transhuman future, as represented by Lucy. This suggests that it is somehow 'natural' for individuals to better themselves, to realize their potential, and thereby draws upon and reproduces the neoliberal view of the autonomous subject who possesses free will and, in taking responsibility for their own fate, acts as their own 'unit of enterprise' (Foucault, 2008: 226).

However, complementing this focus on the neoliberal subject, we have also considered enhancement from the perspective of the broader economic system and the accumulation of 'human capital', to argue that the 'productive body' is an economic entity, shaped in relation to other bodies and technologies and the demands that the system generates. Indeed, organizations have long demanded flexibility on the part of the workforce, to adopt patterns of working according to the demands of the systems of production. In this light, cognitive enhancement might appear as yet another means of proving or realizing one's flexibility to fit with the system, as a way to create the always-on body. ${ }^{18}$ It is no mere accident or contingent matter of biochemistry that the drug modafinil, which is officially prescribed to keep people awake when they are meant to be focused and alert, also appears attractive to those seeking or promoting cognitive enhancement (see, for example, Coveney, 2011; Williams et al., 2008). Preventing inadvertent sleep during the day (narcolepsy), avoiding sleepiness whilst working at night (shift work disorder), avoiding or controlling excessive daytime sleepiness, or seeking to increase focus and alertness on demand (enhancement) all represent efforts to manage the functioning of the brain towards productive or performative ends. Biochemistry may be part of the equation, but so too is the contemporary biopolitical ethos in which the accumulation of capital is increasingly dependent on the accumulation of flexible brains.

\section{Declaration of conflicting interests}

The authors declared no potential conflicts of interest with respect to the research, authorship, and/ or publication of this article. 


\section{Funding}

The authors received no financial support for the research, authorship, and/or publication of this article.

\section{ORCID iD}

Karen Dale (D) https://orcid.org/0000-0001-8881-5375

\section{Notes}

We would like to thank the participants at the symposium on 'Minds and Brains in Everyday Life' in Edinburgh for helpful discussions; Tineke and Susanne for their support and detailed comments on the article; and the anonymous reviewers for their insightful points.

1. This view of plasticity goes well beyond recognizing that the juvenile human brain goesthrough a significant process of development, to emphasize the changes that take place in the brain throughout an individual's whole life course. The concept encompasses the idea of an openness to influences from both within and outside the body, both environmental and - importantly - self-determined, including the effects of brain training, various therapies, harnessing neurofeedback loops, and so on (see, for instance, Brenninkmeijer, 2010), and includes in its effects both individual and potential epigenetic changes. This, then, provides a view of opportunities and threats for the future that focus on the brain (Papadopoulos, 2011; Rose and Abi-Rached, 2013).

2. The term 'smart drugs' is a potentially misleading phrase for a number of reasons. First, in thisuse 'smart' refers to the assumed increase in cognitive capability, but promoting wakefulness or alertness is not the same as the increase of intellect. Second, 'smart drugs' has also been used to describe the search to develop drugs that target and treat only certain symptoms - in other words, the drugs themselves rather than the outcomes are 'smart'. Third, there are no drugs licensed to be prescribed or marketed as 'smart drugs'. Where drugs are taken for their assumed cognitive effects, these are drugs that are prescribed for other conditions (such as narcolepsy or ADHD) and taken off-prescription.

3. And indeed 'an ill for every pill', with the argument that illness and diseases come to beshaped such that they 'fit' pharmacological substances that have been developed.

4. The articles were identified by utilizing the Nexis database of news publications; our searchterms included 'modafinil' or 'smart drugs' coupled with 'brain'. The sample included the following UK national publications: The Guardian and The Observer, The Times and The Sunday Times, The Independent, The Daily Telegraph and The Sunday Telegraph, the Daily Mail and Mail on Sunday, The Sun, The Mirror and The Sunday Mirror, the Daily Express and Sunday Express, the i, and the Daily Star.

5. It is a matter of public record that Cephalon was reprimanded by the FDA for providingproduct information, reinforced by the behaviour of sales staff, that promoted the drug for general symptoms of sleepiness and fatigue well beyond its official authorization (US Department of Justice, 2008). In other words, the pharmacological manipulation of the brain by individuals who felt tired or insufficiently alert when they wished to be alert and attentive was becoming endorsed as a matter of individual choice. 
6. Although Alan Glynn, the author of the original book that inspired the film (The Dark Fields), is clear that it was an entirely fictional drug. Similarly, the drug from Lucy, CPH4, whilst itself a fictional drug, is claimed by the film's director Luc Besson to be based upon a real chemical that is produced in pregnancy to promote the growth and development of the foetus. Web discussions show that individuals have sought for this substance online and sometimes unscrupulous retailers will claim to sell it, whilst others claim that it is modafinil that is the real source of the fictional drug.

7. Psychiatric News (2007) 42(7): 15, available at: https://psychnews.psychiatryonline.org/doi/ pdf/10.1176/pn.2007.42.issue-7.

8. Adderall is a mixture of amphetamine salts, primarily used to treat ADHD.

9. Nootropics are drugs, supplements, and nutritional products that are claimed to improveaspects of mental function (such as memory, motivation, and attention). The term was coined by Corneliu Giurgea from Greek words meaning 'mind' and 'to bend or turn'.

10. https://metro.co.uk/2016/06/01/smart-drugs-are-coming-to-the-office-to-make-yousharperstronger-better-5917892/ (accessed 20th November 2019).

11. https://web.archive.org/web/20150215220209/http://modafinilorder.com/modalert-reviews/

12. For a critical response to this study, see Repantis, Maier, and Heuser (2016).

13. Cephalon was acquired by Teva in 2011. 'Shift work disorder' is a medicalization of thedisruptions to their sleep and wake cycles that shift workers commonly experience, including the inability to sleep during the day or 'excessive sleepiness' whilst on night shift. This has not been a prescribed use of the drug in Europe since 2011, when the European Medicines Agency decided that the potential side effects of modafinil were such that shift work disorder should not be included. Direct marketing of pharmaceuticals to consumers is only legally permitted in the USA and New Zealand. The official website for Nuvigil (www.nuvigil.com), the branded version of modafinil that superseded its forerunner Provigil, offered information concerning a variety of conditions associated with excessive sleepiness and for which the drug might be prescribed. It suggested that some 15 million Americans work outside the 9 to 5 regimen of other employees, and that up to $25 \%$ of them might have SWD.

14. 'Checking Beds, Ready for Her Own' (2012, 1 July) Medical Marketing \& Media, available at: https://www.mmm-online.com/home/channel/features/100-agencies-draftfcb-healthcare/; see also, Pharmacy Today (2013, February: 19).

15. Of course, in accordance with our earlier discussion of morphing and representations ofenhancement, this is not to suggest that modafinil might counter the other deleterious impacts that shift work has on the body - for which there is a growing amount of evidence. Rather, in a sense the imaginary on offer presents the bodily consequences of shift work as merely one of excessive sleepiness, thereby potentially diverting attention away from its more serious health effects.

16. An example of the discussion of this can be seen in Fleming (2017).

17. That said, there is continued online discussion of the merits of the drug for this purpose.

18. One possible further socio-economic factor in the future potential demand and take-up ofcognitive enhancers stems from the increasing automation of work and the substitution of human jobs by technology. 


\section{References}

Abraham, J. (2010) 'Pharmaceuticalization of Society in Context: Theoretical, Empirical and Health Dimensions', Sociology 44(4): 603-22.

Academy of Medical Sciences (2012) Human Enhancement and the Future of Work: Report From a Joint Workshop Hosted by the Academy of Medical Sciences, the British Academy, the Royal Academy of Engineering and the Royal Society, available at: https://acmedsci.ac.uk/policy/ policy/human-enhancement-and-the-future-of-work.

Battleday, R. M. and Brem, A. K. (2015) 'Modafinil for Cognitive Neuroenhancement in Healthy Non-Sleep-Deprived Subjects: A Systematic Review', European Neuropsychopharmacology 25(11): 1865-81.

Beaconsfield, P. (1980) ‘A Pill for Every Ill', The Lancet 315(8163): 315.

Bloomfield, B. and Dale, K. (2015) 'Fit for Work? Redefining "Normal" and "Extreme" Through Human Enhancement Technologies', Organization 22(4): 552-69.

Bloomfield, B. P. and Doolin, B. (2011) 'Imagination and Technoscientific Innovations:

Governance of Transgenic Cows in New Zealand', Social Studies of Science 41(1): 59-83.

British Medical Association (2007) Boosting Your Brainpower: Ethical Aspects of Cognitive Enhancements. London: BMA.

Brenninkmeijer, J. (2010) 'Taking Care of One's Brain: How Manipulating the Brain Changes People's Selves', History of the Human Sciences 23(1): 107-26.

Busfield, J. (2010) “"A Pill for Every Ill”: Explaining the Expansion in Medicine Use', Social Science \& Medicine 70(6): 934-41.

Cadwalladr, C. (2015, 15 February) 'Students Used to Take Drugs to Get High. Now They Take Them to Get Higher Grades', The Observer, available at: https://www.theguardian.com/soci ety/2015/feb/15/students-smart-drugs-higher-grades-adderall-modafinil.

Clay, X. (2012, 12 August) 'Drug-Taking: Think What We'd Achieve If We Never Slept a Wink', The Telegraph, available at: https://www.telegraph.co.uk/news/health/9503763/Drugtakingthink-what-wed-achieve-ifwe-never-slept-a-wink.html.

Cooper, M. (2011) Life as Surplus: Biotechnology and Capitalism in the Neoliberal Era. Seattle: University of Washington Press.

Costea, B., Crump, N., and Amiridis, K. (2007) 'Managerialism and "Infinite Human Resourcefulness": A Commentary Upon the "Therapeutic Habitus", "Derecognition of Finitude" and the Modern Sense of Self', Journal for Cultural Research 11(3): 245-64.

Coveney, C. M. (2011) 'Cognitive Enhancement? Exploring Modafinil Use in Social Context', in M. Pickersgill and I. Van Keulen (eds) Sociological Reflections on the Neurosciences. Bingley: Emerald, pp. 203-28.

Coveney, C., Gabe, J., and Williams, S. (2011) 'The Sociology of Cognitive Enhancement: Medicalisation and Beyond', Health Sociology Review 20(4): 381-93.

Coveney, C. M., Nerlich, B., and Martin, P. (2009) 'Modafinil in the Media: Metaphors, Medicalisation and the Body', Social Science \& Medicine 68(3): 487-95.

Crary, J. (2013) 24/7: Late Capitalism and the Ends of Sleep. London: Verso.

Dale, K. (2001) Anatomising Embodiment and Organisation Theory. Basingstoke: Palgrave.

Dale, K. (2012) 'The Employee as "Dish of the Day": The Ethics of the Consuming/Consumed Self in Human Resource Management', Journal of Business Ethics 111(1): 13-24. 
Dale, K. and Burrell, G. (2014) 'Being Occupied: An Embodied Re-reading of Organizational "Wellness", Organization 21(2): 159-77.

Dawney, L. (2011) 'Social Imaginaries and Therapeutic Self-Work: The Ethics of the Embodied Imagination', The Sociological Review 59(3): 535-52.

Dean, J. (2013, 22 November) 'In the City That Never Sleeps...Traders Stay Up on “Smart” Drugs', The Times, available at: https://www.thetimes.co.uk/article/in-the-city-that-neversleepstraders-stay-up-on-smart-drugs-bg2cts90rd8.

Delgado, A., Rommetveit, K., Barcelo', M., and Lemkow, L. (2012) 'Imagining High-Tech Bodies:

Science Fiction and the Ethics of Enhancement', Science Communication 34(2): 200-40.

Dietz, P., Soyka, M., and Franke, A. G. (2016) 'Pharmacological Neuroenhancement in the Field of Economics - Poll Results From an Online Survey’, Frontiers in Psychology 7: 1-8.

Diver, T. (2017, 24 June) 'Let Students Use Study Drugs and Let's Sell Them at Boots, Says Cambridge Scientist', The Independent, available at: http://www.independent.co.uk/student/ news/let-students-use-study-drugs-modafinil-narcotics-sell-at-boots-top-cambridgescientistbarbara-a7806111.html.

Elliott, C. (2003) Better Than Well: American Medicine Meets the American Dream. New York: W. W. Norton.

Farah, M. J., Illes, J., Cook-Deegan, R., Gardner, H., Kandel, E., King, P., Parens, E., Sahakian, B., and Wolpe, P. R. (2004) 'Neurocognitive Enhancement: What Can We Do and What Should We Do?', Nature Reviews (Neuroscience) 5(5): 421-5.

Farr, M. (2014, 27 May) 'Public Servants Used Drug, Modafinil, to Stay Awake to Complete the Federal Budget on Time', News.com.au, available at: https://www.news.com.au/national/pub lic-servants-used-drug-modafinil-to-stay-awake-to-complete-the-federal-budget-on-time/ news-story/8bc517ef259fbfbb882ca68b3bd405a4.

Featherstone, M. (1991) 'The Body in Consumer Culture', in M. Featherstone, M. Hepworth, and B. S. Turner (eds) The Body: Social Process and Cultural Theory. London: SAGE, pp. 170-96.

Fleming, N. (2017, 30 January) 'Is It Safe to Take Melatonin for Jet Lag?', The Guardian, available at: https://www.theguardian.com/lifeandstyle/2017/jan/30/is-it-safe-to-use-melato nin-for-jet-lag-insomnia.

Fleming, P. (2015) The Mythology of Work: How Capitalism Persists Despite Itself. London: Pluto Press.

Flew, T. (2014) 'Six Theories of Neoliberalism', Thesis Eleven 122(1): 49-71.

Foucault, M. (1977) Discipline and Punish: The Birth of the Prison, trans. A. Sheridan. London: Penguin.

Foucault, M. (2008) The Birth of Biopolitics: Lectures at the Colle'ge de France, 1978-1979, ed. M. Senellart, trans. G. Burchell. Basingstoke: Palgrave Macmillan.

Fox, N. J. and Ward, K. J. (2008) 'Pharma in the Bedroom ....and the Kitchen. ...The Pharmaceuticalisation of Daily Life', Sociology of Health \& Illness 30(6): 856-68.

Frosch, D. L., Krueger, P. M., Hornik, R. C., Cronholm, P. F., and Barg, F. K. (2007) 'Creating Demand for Prescription Drugs: A Content Analysis of Television Direct-to-Consumer Advertising', Annals of Family Medicine 5(1): 6-13.

Gatens, M. (1996) Imaginary Bodies: Ethics, Power and Corporeality. London: Routledge. 
Greely, H., Sahakian, B., Harris, J., Kessler, R. C., Gazzaniga, M., Campbell, P., and Farah, M. J. (2008) 'Towards Responsible Use of Cognitive-Enhancing Drugs by the Healthy', Nature 456(7223): 702-5.

Gue'ry, F. and Deleule, D. (2014) The Productive Body, ed. and trans. P. Barnard and S. Shapiro. Alresford: Zero Books.

Harvey, D. (1998) 'The Body as an Accumulation Strategy', Environment and Planning D: Society and Space 16(4): 401-21.

Harvey, D. and Haraway, D. (1995) 'Nature, Politics, and Possibilities: A Debate and Discussion With David Harvey and Donna Haraway', Environment and Planning D: Society and Space 13(5): 507-27.

Hogle, L. F. (2005) 'Enhancement Technologies and the Body', Annual Review of Anthropology 34: $695-716$.

Hollway, W. (1991) Work Psychology and Organizational Behaviour: Managing the Individual at Work. London: SAGE.

'How (and Why) to Use Nootropics to Boost Productivity and Performance' (2016, 22 December) MoneyMiniBlog, available at: https://moneyminiblog.com/productivity/smart-drugs/.

Joelving, F. (2011, 2 November) 'Modafinil Has Mixed Effects on Dozy Surgeons', Reuters, available at: https://uk.reuters.com/article/health-us-modafinil/modafinil-has-mixedeffectson-dozy-surgeons-idUKTRE7A17ZU20111102.

Karim, F. (2017, 30 December) 'A Drop of LSD Is "New Brain Booster", The Times, available at: https://www.thetimes.co.uk/article/a-drop-of-lsd-is-new-brain-booster-w2kbtw559.

Kroll-Smith, S. (2003) 'Popular Media and "Excessive Daytime Sleepiness": A Study of Rhetorical Authority in Medical Sociology', Sociology of Health \& Illness 25(6): 625-43.

Krueger, G. P. and Leaman, H. M. (2011) Effects of Psychoactive Chemicals on Commercial Driver Health and Performance: Stimulants, Hypnotics, Nutritional, and Other Supplements. Washington, DC: Transportation Research Board, available at: http://onlinepubs.trb.org/onli nepubs/ctbssp/ctbssp_syn_19.pdf.

Kuchler, H. (2017, 10 August) 'How Silicon Valley Rediscovered LSD', Financial Times, available at: https://www.ft.com/content/0a5a4404-7c8e-11e7-ab01-a13271d1ee9c.

Laurance, J. (1995, 30 October) 'Mechanical heart hastens end of human transplants', The Times.

Lay, K. (2015, 20 August) 'Smart Drug Helps You to Sleep Less and Think More', The Times, available at: https://www.thetimes.co.uk/tto/health/news/article4532339.ece.

Le Doeuff, M. (1989) The Philosophical Imaginary, trans. C. Gordon. London: Athlone.

Lemke, T. (2002) 'Foucault, Governmentality, and Critique', Rethinking Marxism 14(3): 49-64.

Louv, J. (2012, 12 December) 'How Smart Drugs and Cybernetics Could Create a Superhuman Workforce', Motherboard, available at: https://www.vice.com/en_us/article/gvvy9y/howsmartdrugs-and-cybernetics-could-create-a-superhuman-workforce.

Macnaghten, P., Kearnes, M. B., and Wynne, B. (2005) 'Nanotechnology, Governance, and Public Deliberation: What Role for the Social Sciences?', Science Communication 27(2): 268-91.

Margo, J. (2000, 24 June) 'Wake to a Bright New Day/STAYING ALIVE', The Weekend Australian, p. R31. 
Martin, E. (1994) Flexible Bodies: Tracking Immunity in American Culture From the Days of Polio to the Age of AIDS. Boston: Beacon Press.

Martin, E. (2006) 'The Pharmaceutical Person', BioSocieties 1(3): 273-87.

Moreno, J. D. (2008) Mind Wars: Brain Science and the Military in the 21st Century. New York: Bellevue Literary Press.

'Nootropics: Can These Smart Drugs Super-Charge Your Career?' (2013, 16 May) Fox News, available at: https://www.foxnews.com/health/nootropics-can-these-smart-drugs-superchargeyour-career.

O'Connor, C. and Joffe, H. (2015) 'How the Public Engages With Brain Optimization: The MediaMind Relationship', Science, Technology, \& Human Values 40(5): 712-43.

Papadopoulos, D. (2011) 'The Imaginary of Plasticity: Neural Embodiment, Epigenetics and Ecomorphs', The Sociological Review 59(3): 432-56.

Petrow, S. (2013, 4 November) 'The Drugs of Work-Performance Enhancement', The Atlantic, available at: https://www.theatlantic.com/health/archive/2013/11/the-drugs-of-work-perfor mance-enhancement/281055/.

'The PharmaceuticalPathto a SuperhumanWorkforce'(2012,23 November)Financial Times, p. 12.

Pitts-Taylor, V. (2010) 'The Plastic Brain: Neoliberalism and the Neuronal Self', Health 14(6): 635-52.

Pollack, A. (2010, 29 March) 'Regulators Reject a Drug Maker's Plan to Use Its Alertness Pill to Overcome Jet Lag', New York Times, available at: https:/www.nytimes.com/2010/03/30/busi ness/30drug.html?_r1\%40.

Rabinbach, A. (1992) The Human Motor: Energy, Fatigue, and the Origins of Modernity. Berkeley: University of California Press.

Racine, E. and Forlini, C. (2010) 'Cognitive Enhancement, Lifestyle Choice or Misuse of Prescription Drugs? Ethics Blind Spots in Current Debates', Neuroethics 3(1): 1-4.

Rasmussen, N. (2008) On Speed: The Many Lives of Amphetamine. New York: New York University Press.

Repantis, D., Maier, L. J., and Heuser, I. (2016) 'Correspondence Arising: Modafinil for Cognitive Neuroenhancement in Health [sic] Non-Sleep-Deprived-Subjects', European Neuropsychopharmacology 26(2): 392-3.

Repantis, D., Schlattmann, P., Laisney, O., and Heuser, I. (2010) 'Modafinil and Methylphenidate for Neuroenhancement in Healthy Individuals: A Systematic Review', Pharmacological Research 62(3): 187-206.

Roose, K. (2015, 4 March) 'I Tried Silicon Valley's Favorite “Brain-Enhancing” Drugs', Splinter, available at: https://splinternews.com/i-tried-silicon-valleys-favorite-brain-enhancingdrugs 1793845948.

Rose, N. (2007) 'Molecular Biopolitics, Somatic Ethics and the Spirit of Biocapital', Social Theory \& Health 5(1): 3-29.

Rose, N. and Abi-Rached, J. M. (2013) Neuro: The New Brain Sciences and the Management of the Mind. Princeton: Princeton University Press.

Sawday, J. (1995) The Body Emblazoned: dissection and the human body in Renaissance culture

London: Routledge. 
Sharma, J. (2004, 14 July) 'Sleepless in the City', Times of India, available at: https://timesofindia. indiatimes.com/delhi-times/Sleepless-in-the-city/articleshow/777288.cms.

Singh, I. (2007) 'Not Just Naughty: 50 Years of Stimulant Drug Advertising', in A. Tone and E. Siegel Watkins (eds) Medicating Modern America: Prescription Drugs in History. New York: New York University Press, pp. 131-55.

Smith, C. and Land, C. (2014) 'Pharmacological Routes to Everyday Exceptionality', Culture and Organization 20(4): 269-87.

Smith, C. and Riach, K. (2016) 'Drug Taking and Employment: Exploring the Employable Citizen in UK Policy’, Sociology 50(1): 24-42.

Sobchack, V., ed. (2000) Meta Morphing: Visual Transformation and the Culture of QuickChange. Minneapolis: University of Minnesota Press.

Sugden, C., Housden, C. R., Aggarwal, R., Sahakian, B., and Darzi, A. (2012) 'Effect of Pharmacological Enhancement on the Cognitive and Clinical Psychomotor Performance of Sleep-Deprived Doctors: A Randomized Controlled Trial', Annals of Surgery 255(2): 222-7.

Talbot, M. (2009, 20 April) 'Brain Gain: The Underground World of "Neuroenhancing” Drugs', New Yorker, available at: https://www.newyorker.com/magazine/2009/04/27/brain-gain.

Taylor, C. (2002) 'Modern Social Imaginaries', Public Culture 14(1): 91-124.

Thomson, H. (2015, 20 August) 'Narcolepsy Medication Modafinil Is World's First Safe "Smart Drug", The Guardian, available at: https://www.theguardian.com/science/2015/aug/20/narco lepsy-medication-modafinil-worlds-first-safe-smart-drug.

Tone, A. (2009) The Age of Anxiety: A History of America's Turbulent Affair With Tranquilizers. New York: Basic Books.

Turner, B. S. (1984) The Body and Society: Explorations in Social Theory. Oxford: Basil Blackwell.

US Department of Justice (2008, 29 September) 'Biopharmaceutical Company, Cephalon, to Pay \$425 Million \& Enter Plea to Resolve Allegations of Off-Label Marketing', available at: https://www.justice.gov/archive/opa/pr/2008/September/08-civ-860.html.

Vercellone, C. (2005, November) 'The Hypothesis of Cognitive Capitalism', paper presented at the Historical Materialism Annual Conference, Birkbeck College and SOAS, London, available at: https://hal-paris1.archives-ouvertes.fr/halshs-00273641/.

Vrecko, S. (2013) 'Just How Cognitive Is "Cognitive Enhancement”? On the Significance of Emotions in University Students' Experiences With Study Drugs', AJOB Neuroscience 4(1): 4-12.

Vrecko, S. (2015) 'Everyday Drug Diversions: A Qualitative Study of the Illicit Exchange and Non-Medical Use of Prescription Stimulants on a University Campus', Social Science \& Medicine 131: 297-304.

Williams, S. J., Coveney, C. M., and Gabe, J. (2013) 'Medicalisation or Customisation? Sleep, Enterprise and Enhancement in the 24/7 Society', Social Science \& Medicine 79: 40-7.

Williams, S. J., Seale, C., Boden, S., Lowe, P., and Steinberg, D. L. (2008) 'Waking Up to Sleepiness: Modafinil, the Media and the Pharmaceuticalisation of Everyday/Night Life', Sociology of Health \& Illness 30(6): 839-55. 
Wolf-Meyer, M. (2009) 'Precipitating Pharmakologies and Capital Entrapments: Narcolepsy and the Strange Cases of Provigil and Xyrem', Medical Anthropology 28(1): 11-30.

Wolf-Meyer, M. J. (2012) The Slumbering Masses: Sleep, Medicine, and Modern American Life. London: University of Minnesota Press.

Woollaston, V. (2014, 31 March) 'The Real-Life Limitless Pill? Drug Helps Adults Learn as Fast as Children by Making the Brain More "Elastic", Mail Online, available at: http://www.dailymail.co.uk/sciencetech/article-2593268/The-drug-helps-adults-learnfastchildren.html.

\section{Author biographies}

Brian P. Bloomfield is Professor Emeritus in the Department of Organisation, Work and Technology at Lancaster University, UK. His research interests include social imaginaries and technoscientific innovations, debates about human enhancement technologies, and posthumanism. He has published in the fields of organization studies, sociology, and science and technology studies.

Karen Dale is Professor of Organisation Studies in the Department of Organisation, Work and Technology at Lancaster University, UK. She has written about embodiment, architecture, space, and social materiality related to organization studies. Her current research is into performance enhancing drugs at work, and includes reports for the European Agency for Safety and Health at Work. 